\title{
Longitudinal filament oscillations enhanced by two C-class flares ${ }^{\star}$
}

\author{
Q. M. Zhang ${ }^{1,2,3}$, J. H. Guo ${ }^{3,4}$, K. V. Tam ${ }^{2}$, and A. A. $\mathrm{Xu}^{2}$ \\ 1 Key Laboratory of Dark Matter and Space Science, Purple Mountain Observatory, CAS, Nanjing 210033, PR China \\ e-mail: zhangqm@pmo.ac.cn \\ 2 State Key Laboratory of Lunar and Planetary Sciences, Macau University of Science and Technology, Macau, PR China \\ 3 School of Astronomy and Space Science, Nanjing University, Nanjing 210023, PR China \\ ${ }^{4}$ Key Laboratory of Modern Astronomy and Astrophysics (Nanjing University), Ministry of Education, Nanjing 210023, PR China
}

Received 10 December 2019 / Accepted 5 January 2020

\begin{abstract}
Context. Large-amplitude, longitudinal filament oscillations triggered by solar flares have been well established in the literature. However, filament oscillations enhanced by flares have never been reported.

Aims. In this paper we report the multiwavelength observations of a very long filament in active region (AR) 11112 on 2010 October 18. The filament was composed of two parts, the eastern part (EP) and the western part (WP). We focus on longitudinal oscillations of the EP, which were enhanced by two homologous C-class flares in the same AR.

Methods. The filament was observed in $\mathrm{H} \alpha$ wavelength by the Global Oscillation Network Group and in extreme ultraviolet wavelengths by the Atmospheric Imaging Assembly on board the Solar Dynamics Observatory (SDO). Line-of-sight magnetograms were provided by the Helioseismic and Magnetic Imager on board SDO. The global three-dimensional magnetic fields were obtained using the potential field source surface modeling. Soft X-ray light curves of the two flares were recorded by the GOES spacecraft. White light images of the corona were observed by the LASCO/C2 coronagraph on board SOHO. To reproduce part of the observations, we perform one-dimensional, hydrodynamic numerical simulations using the MPI-AMRVAC code.

Results. The C1.3 flare was confined without a coronal mass ejection (CME). Both EP and WP of the filament were slightly disturbed and survived the flare. After $5 \mathrm{~h}$, eruption of the WP generated a C2.6 flare and a narrow jet-like CME. Three oscillating threads $\left(\operatorname{thd}_{a}, \operatorname{thd}_{b}, \mathrm{hd}_{c}\right.$ ) are obviously identified in the EP, and their oscillations are naturally divided into three phases by the two flares. The initial amplitude ranges from 1.6 to $30 \mathrm{Mm}$ with a mean value of $\sim 14 \mathrm{Mm}$. The period ranges from 34 to 73 min with a mean value of $\sim 53 \mathrm{~min}$. The curvature radii of the magnetic dips are estimated to be 29 to $133 \mathrm{Mm}$ with a mean value of $\sim 74 \mathrm{Mm}$. The damping times ranges from $\sim 62$ to $\sim 96 \mathrm{~min}$ with a mean value of $\sim 82 \mathrm{~min}$. The value of $\tau / P$ is between 1.2 and 1.8 . For thd ${ }_{a}$ in the EP, the amplitudes were enhanced by the two flares from $6.1 \mathrm{Mm}$ to $6.8 \mathrm{Mm}$ after the $\mathrm{C} 1.3$ flare, and further to $21.4 \mathrm{Mm}$ after the $\mathrm{C} 2.6$ flare. The period variation as a result of perturbation from the flares was within $20 \%$. The attenuation became faster after the C2.6 flare. Conclusions. To the best of our knowledge, this is the first report of large-amplitude, longitudinal filament oscillations enhanced by flares. Numerical simulations reproduce the oscillations of thd $\mathrm{d}_{a}$ very well. The simulated amplitudes and periods are close to the observed values, while the damping time in the last phase is longer, implying additional mechanisms should be taken into account apart from radiative loss.
\end{abstract}

Key words. Sun: coronal mass ejections (CMEs) - Sun: filaments, prominences - Sun: flares - Sun: oscillations - methods: numerical

\section{Introduction}

Solar prominences are dense and cool plasma structures suspending in the corona (Labrosse et al. 2010; Mackay et al. 2010; Parenti 2014; Vial \& Engvold 2015; Gibson 2018, and references therein). The density of prominences is two orders of magnitude higher than the corona, while the temperature is two orders of magnitude lower than the corona. Prominences are also called filaments that appear darker than the surroundings on the solar disk (Engvold 1976; Martin 1998). Prominences (or filaments) can be observed in $\mathrm{H} \alpha$, CaII H, He I $10830 \AA$, extremeultraviolet (EUV), and radio wavelengths (e.g., van Ballegooijen 2004; Berger et al. 2010; Schmieder et al. 2010, 2014; Shen et al. 2015; Yan et al. 2015; Yang et al. 2017). It is generally accepted that the gravity of prominences is balanced by the upward tension force of magnetic dips in sheared arcades (Xia et al. 2012; Keppens \& Xia 2014; Gunár \& Mackay 2015), magnetic flux ropes (DeVore \& Antiochos 2000; Martens \& Zwaan 2001; Su

\footnotetext{
* Movies associated to Figs. 3, 4, and 14 are available at https://www . aanda.org
}

\& van Ballegooijen 2012), or both (Liu et al. 2012). Filaments are located in filament channels in active regions (ARs), quiet regions, and polar regions. The origins of material in filaments include direct injection from the chromosphere (Li \& Zhang 2013), upward elevation from below (Lites 2005), evaporationcondensation (Xia et al. 2011), and reconnection-condensation (Li et al. 2018a). The lifetimes of filaments range from a few hours to several days. After destabilization, they are likely to erupt and generate flares and/or coronal mass ejections (CMEs; Shibata et al. 1995). When the compression from the large-scale overlaying magnetic field lines is strong enough, the filament fails to erupt successfully and evolves into a CME (Ji et al. 2003; Zhang et al. 2015).

After being disturbed, filaments are prone to oscillate periodically (Oliver \& Ballester 2002; Arregui \& Ballester 2011; Arregui et al. 2012). The period of oscillations ranges from a few minutes to tens of minutes or even hours (Jing et al. 2006; Lin et al. 2007). In most cases they oscillate with small amplitudes and short periods of 3-5 min (Ning et al. 2009; Li et al. 2018b). Large-amplitude oscillations are often observed as a 
result of sudden attacks, such as flares (Ramsey \& Smith 1966), microflares (Jing et al. 2003; Vršnak et al. 2007), coronal jets (Luna et al. 2014; Zhang et al. 2017a), surges (Chen et al. 2008), shock waves (Shen et al. 2014a; Pant et al. 2016), and global EUV waves associated with CMEs (Eto et al. 2002; Shen et al. 2014b).

Due to various kinds of disturbances, the direction of filament oscillations may change from case to case (Luna et al. 2018). For longitudinal oscillations, the filament material oscillates along the threads with small angles of $10^{\circ}-20^{\circ}$ between the threads and spine (Jing et al. 2003; Vršnak et al. 2007; Zhang et al. 2012; Li \& Zhang 2012; Bi et al. 2014; Chen et al. 2014; Luna et al. 2014, 2017; Zhou et al. 2018; Awasthi et al. 2019; Zapiór et al. 2019). The polarization of transverse oscillations could be horizontal (vertical) if the whole body oscillates parallel (vertical) to the solar surface (Hyder 1966; Kleczek \& Kuperus 1969; Zhang \& Ji 2018). Sometimes two filaments experience different types of oscillations due to different angles between the incoming waves and filaments (Shen et al. 2014a). Occasionally, different parts of a whole filament experience different types of oscillations (Pant et al. 2016; Zhang et al. 2017a; Mazumder et al. 2020). Interestingly, the parameters of oscillations, including amplitude, period, and damping time vary with time, possibly due to the thread-thread interaction (Zhang et al. 2017b; Zhou et al. 2017). Hence, filament oscillations show complex and complicated behaviors.

The primary restoring force of large-amplitude longitudinal filament oscillations is believed to be the gravity of filament (Luna \& Karpen 2012; Luna et al. 2012, 2016; Zhang et al. 2012, 2013). Therefore, longitudinal oscillations can be described excellently with a pendulum model. Curvature radii of magnetic dips can be exactly diagnosed, and the lower limits of transverse magnetic field strength of the dips can be roughly estimated (Luna et al. 2014, 2017; Zhang et al. 2017b,a; Mazumder et al. 2020). The damping mechanisms, however, are still controversial. Hydrodynamic (HD) and magnetohydrodynamic (MHD) numerical simulations have shed light on the damping mechanisms, such as mass accretion (Luna \& Karpen 2012; Ruderman \& Luna 2016), radiative loss (Zhang et al. 2013), and wave leakage (Zhang et al. 2019). When mass drainage takes place at the footpoints of coronal loops as a result of large initial amplitudes, the damping times are significantly reduced (Zhang et al. 2013).

To date, longitudinal filament oscillations triggered by flares have been extensively investigated. However, filament oscillations enhanced by flares have never been reported. In this article we report the multiwavelength observations of longitudinal oscillations of a filament in AR 11112 on 2010 October 18. The evolution of filament oscillations was divided into three phases, during which a confined $\mathrm{C} 1.3$ flare and an eruptive C2.6 flare associated with a jet-like CME occurred in the AR. Data analyses are described in Sect. 2. Observational results are shown in Sect. 3. To reproduce part of the observations, we performed one-dimensional (1D) HD numerical simulations, which are described in Sect. 4. Discussions and a brief summary are presented in Sects. 5 and 6.

\section{Observations and data analysis}

The filament with sinistral chirality was observed in $\mathrm{H} \alpha$ line center by the ground-based telescope of Global Oscillation Network Group (GONG). It was also observed in EUV wavelengths (171 and $304 \AA$ ) by the Atmospheric Imaging Assembly (AIA; Lemen et al. 2012) on board the Solar Dynamics Observatory (SDO). The photospheric line-of-sight (LOS) mag-
Table 1. Description of the observational parameters.

\begin{tabular}{lcccc}
\hline \hline Instrument & $\begin{array}{c}\lambda \\
(\AA)\end{array}$ & $\begin{array}{c}\text { Time } \\
(\mathrm{UT})\end{array}$ & $\begin{array}{c}\text { Cadence } \\
(\mathrm{s})\end{array}$ & $\begin{array}{c}\text { Pixel size } \\
\left({ }^{\prime \prime}\right)\end{array}$ \\
\hline GONG & 6562.8 & $10: 55-22: 00$ & 60 & 1.0 \\
SDO/AIA & 171,304 & $07: 00-22: 00$ & 12 & 0.6 \\
SDO/AIA & 1600 & $07: 00-22: 00$ & 24 & 0.6 \\
SDO/HMI & 6173 & $07: 00-22: 00$ & 45 & 0.6 \\
SDO/EVE & $1-70$ & $07: 00-22: 00$ & 0.25 & $\ldots$ \\
LASCO/C2 & WL & $16: 50-17: 48$ & 720 & 11.4 \\
GOES & $0.5-4$ & $07: 00-22: 00$ & 2.05 & $\ldots$ \\
GOES & $1-8$ & $07: 00-22: 00$ & 2.05 & $\ldots$ \\
\hline
\end{tabular}

netograms were provided by the Helioseismic and Magnetic Imager (HMI; Scherrer et al. 2012) on board SDO. The AIA and HMI data were calibrated using the standard Solar Software (SSW) programs aia_prep.pro and hmi_prep.pro. The fulldisk $\mathrm{H} \alpha$ and AIA $304 \AA$ A images were coaligned with a precision of $\sim 1$ ".2 using the cross-correlation method. Three-dimensional (3D) global magnetic fields at 12:04 UT were obtained by the potential field source surface (PFSS; Schrijver \& De Rosa 2003) modeling. The 1-70 $\AA$ flux of the flares was recorded by the Extreme Ultraviolet Variability Experiment (EVE; Woods et al. 2012) on board SDO. Soft X-ray (SXR) fluxes of the flares in $0.5-4 \AA$ and $1-8 \AA$ were recorded by the GOES spacecraft. The CME associated with the C2.6 flare was observed by the C2 white light (WL) coronagraph of the Large Angle Spectroscopic Coronagraph (LASCO; Brueckner et al. 1995) on board $\mathrm{SOHO}^{1}$. The LASCO/C2 data were calibrated using the SSW program c2_calibrate.pro. The observational parameters from multiple instruments are listed in Table 1.

\section{Observational results}

\subsection{Confined flare}

In Fig. 1 the top panels show 171 and $304 \AA$ images observed by AIA at 07:00 UT. The white arrows point to the long dark filament residing in AR 11112. Panel c shows the photospheric LOS magnetogram observed by HMI at 06:59 UT. Panel d shows the $\mathrm{H} \alpha$ image observed by GONG at 10:55:34 UT. It is clear that the filament is located along the polarity inversion line of the AR and is divided into two parts. The eastern part (EP) has an apparent length of $\sim 650^{\prime \prime}$ and an angle of $\sim 26^{\circ}$ relative to the EW direction. The western part (WP) has an apparent length of $\sim 250^{\prime \prime}$ and an angle of $\sim 47^{\circ}$ relative to the EW direction. Hence, the total length of filament reaches $\sim 900^{\prime \prime}$. After correcting the projection effect, the true length of the filament is close to the solar radius $\left(\sim 960^{\prime \prime}\right)$.

During the whole evolution of the filament, two homologous flares occurred in the AR. The first was a confined C1.3 flare. In Fig. 2 SXR light curves in $0.5-4$ and $1-8 \AA$ during 07:00-22:00 UT are plotted in panel a. SXR intensities of the flare started to increase at $\sim 11: 11$ UT and reached the peak values at $\sim 11: 39$ UT before decreasing gradually until $\sim 12: 24$ UT. The time derivative of the $1-8 \AA$ flux as a rough proxy of the hard X-ray (HXR) flux is plotted in panel b, where the first black dashed line denotes the peak time at $\sim 11: 33: 20$ UT. Panel $\mathrm{c}$ shows the EVE 1-70 $\AA$ light curve with a similar evolution to the $1-8 \AA$ light curve except there are delayed peaks.

1 http://cdaw.gsfc.nasa.gov/CME_list/ 


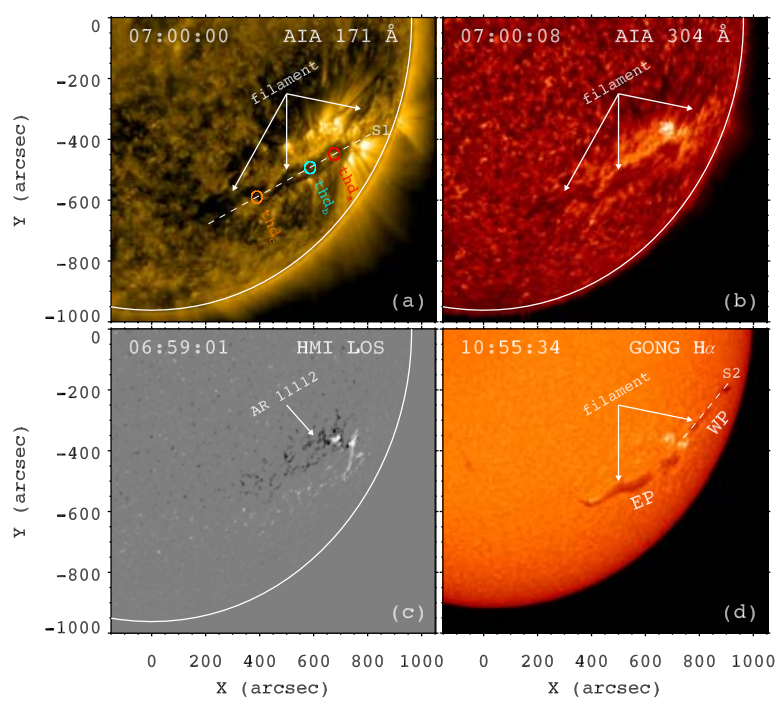

Fig. 1. Panels $a-b$ : AIA 171 and $304 \AA$ images at 07:00 UT. The arrows point to the long filament. Panel $c$ : HMI LOS magnetogram at 06:59 UT. The arrow points to AR 11112. Panel $d$ : GONG H $\alpha$ image at 10:55:34 UT. "EP" and "WP" represent the eastern and western parts of the filament, respectively. In panel $a$ a slice (S1) is selected to investigate the longitudinal oscillations of EP, whose time-distance diagram is shown in Fig. 10. The red, cyan, and orange circles indicate the oscillating threads $\left(\right.$ thd $_{a}$, thd $_{b}$, and thd $d_{c}$ ) in EP. In panel $d$ a slice (S2) is selected to investigate the evolution of WP, whose time-distance diagram is shown in Fig. 5.

\subsection{Eruptive flare and jet-like CME}

About $5 \mathrm{~h}$ later, the $\mathrm{C} 2.6$ flare took place in the same AR. Figure 2a shows that SXR intensities of the flare start to increase at $\sim 16: 15$ UT and reach the peak values at $\sim 16: 42$ UT before decreasing slowly until $\sim 18: 25$ UT. Figure 2 b shows two HXR peaks at 16:31:30 UT and 16:37:30 UT that are associated with the flare. Figure 4 shows the AIA 171, 304, $1600 \AA$, and GONG $\mathrm{H} \alpha$ images around 16:33 UT. The flare ribbon is cospatial with the main ribbon of the $\mathrm{C} 1.3$ flare. It is found that the ribbons of both flares are located near the two endpoints of the EP.

Figure 3 shows the AIA 171, 304, $1600 \AA$, and GONG H $\alpha$ images around 11:34 UT. Bright flare ribbons close to the filament are evident in all wavelengths. Since the flare was confined, both EP and WP of the filament were slightly disturbed without erupting into a CME.

Interestingly, the EP remained there and did not erupt during this flare, while the WP disappeared (see Fig. 4d). In Fig. 1d an artificial slice (S2) along the WP with a length of $265^{\prime \prime}$ is selected to investigate its evolution. The time-slice diagram of $\mathrm{S} 2$ in $\mathrm{H} \alpha$ is displayed in Fig. 5. It is obvious that the WP was slightly disturbed during the $\mathrm{C} 1.3$ flare, while it was strongly disturbed during the $\mathrm{C} 2.6$ flare and finally erupted. Intermittent mass flow toward the AR at speeds of $10-20 \mathrm{~km} \mathrm{~s}^{-1}$ before eruption is clearly seen in the diagram.

Eruption of the WP in $304 \AA$ was indicated as jet-like outflow. In Fig. 6, the left panel shows a snapshot of $304 \AA$ image at 16:34:56 UT. An artificial slice (S3) with a length of $363^{\prime \prime}$ is selected to investigate the outflow. The time-slice diagram of S3 in $304 \AA$ is displayed in the right panel. It is seen that the outflow spurted out from $\sim 16: 15$ UT and lasted for about $30 \mathrm{~min}$. The occurrence time of outflow was coincident with the SXR peak time of C2.6 flare. The estimated apparent velocities (310-410 $\mathrm{km} \mathrm{s}^{-1}$ ) are comparable to those of typical coronal jets (Zhang \& Ji 2014).

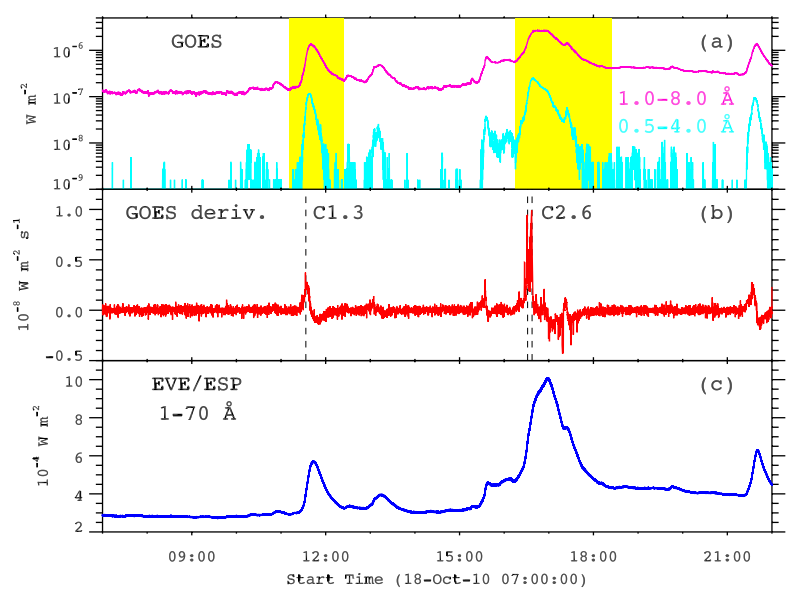

Fig. 2. Panel a: GOES SXR light curves during 07:00-22:00 UT on 2010 October 18 . The yellow regions denote the times of the confined C1.3 flare and eruptive C2.6 flare. Panel $b$ : time derivative of the 1-8 light curve. Panel c: EVE 1-70 A light curve.

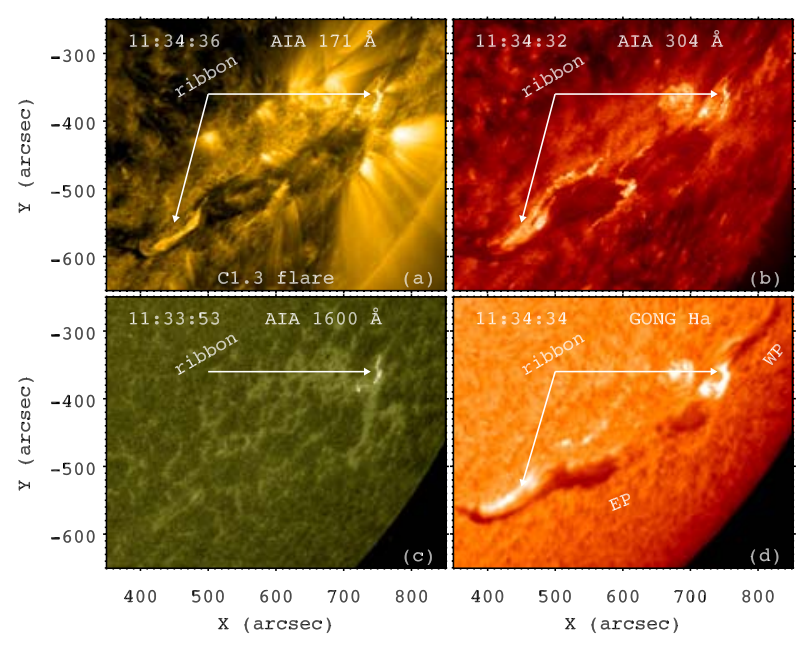

Fig. 3. AIA 171, 304, $1600 \AA$, and GONG H $\alpha$ images around 11:34 UT. The arrows point to the bright flare ribbons. The evolution of the $\mathrm{C} 1.3$ flare is shown in a movie (anim1.avi) available online.

The 3D magnetic field lines around AR 11112 at 12:04 UT are drawn in Fig. 7, with open and closed field lines being coded with green/magenta and white lines, respectively. The direction of the open field (green lines) is consistent with the propagation direction of jet-like outflow, suggesting that the outflow propagated along open field lines.

White light observations of the corona from $\mathrm{LASCO} / \mathrm{C} 2$ indicate that the jet-like outflow propagated even more and evolved into a narrow CME. Figure 8 shows five snapshots of running-difference images observed by LASCO/C2. The CME appeared at 16:59 UT and propagated until 17:48 UT in the southwest direction, which is also consistent with the direction of open field in Fig. 7. The central position angle (CPA) and angular width are $216^{\circ}$ and $10^{\circ}$, respectively. Height-time plot of the CME is displayed in Fig. 9. A linear fitting of the plot results in an apparent velocity of $\sim 750 \mathrm{~km} \mathrm{~s}^{-1}$ in the plane of sky. It should be noted that the measurements of CME heights have significant uncertainties since it is not easy to identify the leading edge. Therefore, the derived velocity of CME is nearly twice as high as that of outflow observed in EUV wavelengths. 


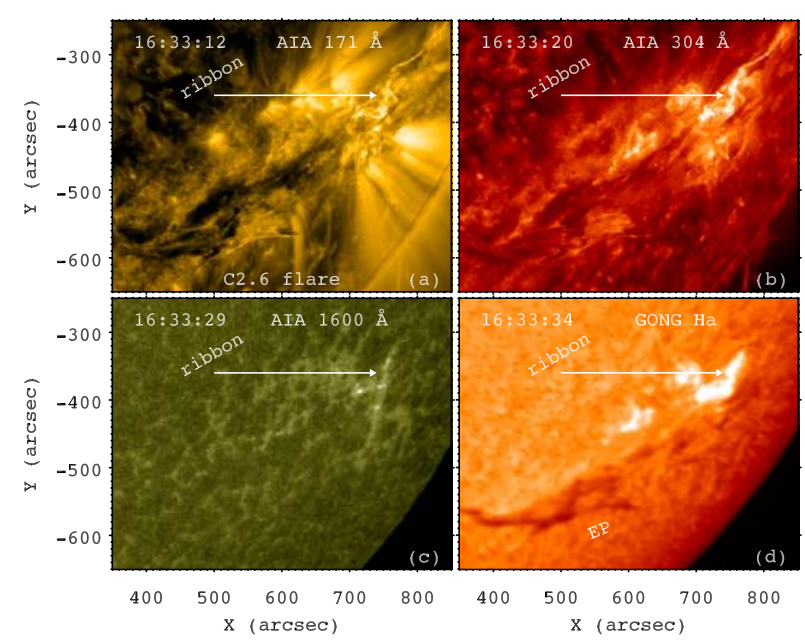

Fig. 4. Same as Fig. 3, but for the C2.6 flare. The evolution of C2.6 flare is shown in a movie (anim2.avi) available online.

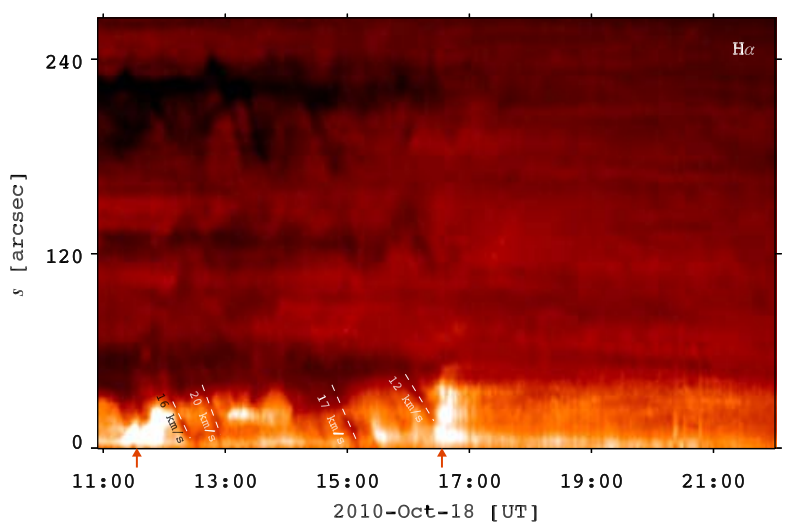

Fig. 5. Time-distance diagram of $\mathrm{S} 2$ in $\mathrm{H} \alpha$. On the $y$-axis, $s=0^{\prime \prime}$ and $s=265^{\prime \prime}$ represent the southeast and northwest endpoints of S2. Two vertical arrows under the $x$-axis indicate the times (11:33 UT and 16:33 UT) of flares. The velocities of mass flow toward the AR are labeled.

\subsection{Filament oscillations}

During 07:00-22:00 UT, the EP oscillated longitudinally along the filament channel. In Fig. 1a, at least three oscillating threads $\left(\right.$ thd $_{a}, \operatorname{thd}_{b}$, and thd ${ }_{c}$ ) could be identified and marked by red, cyan, and orange circles, respectively. An artificial slice (S1) with a length of $669^{\prime \prime}$ is selected to investigate the evolution of EP. The time-slice diagram of S1 in $171 \AA$ is plotted in Fig. 10. It is found that different threads oscillated in a different way, showing a very complex behavior. Six subregions (sub1-sub6) within the cyan boxes are extracted: sub1 and sub2 represent oscillations before the $\mathrm{C} 1.3$ flare; sub3 and sub4 represent oscillations between the two flares; sub5 and sub6 represent oscillations after the C2.6 flare. For thd $\mathrm{d}_{a}$ the oscillation was divided into three phases. Before the $\mathrm{C} 1.3$ flare, it oscillated with a relatively small amplitude and insignificant damping (see sub1). After the C1.3 flare, the amplitude increased slightly (see sub3). After the C2.6 flare, the amplitude became even larger with faster damping (see sub5). For thd $d_{b}$ the oscillation between the two flares was clearly demonstrated, suggesting that the oscillation was triggered by the $\mathrm{C} 1.3$ flare. After the $\mathrm{C} 2.6$ flare, it became chaotic and no distinct oscillation could be recognized. For thd $\mathrm{d}_{c}$ the oscillations were present before the $\mathrm{C} 1.3$ flare and after the $\mathrm{C} 2.6$ flare. Between the two flares, the oscillation was not so obvious.
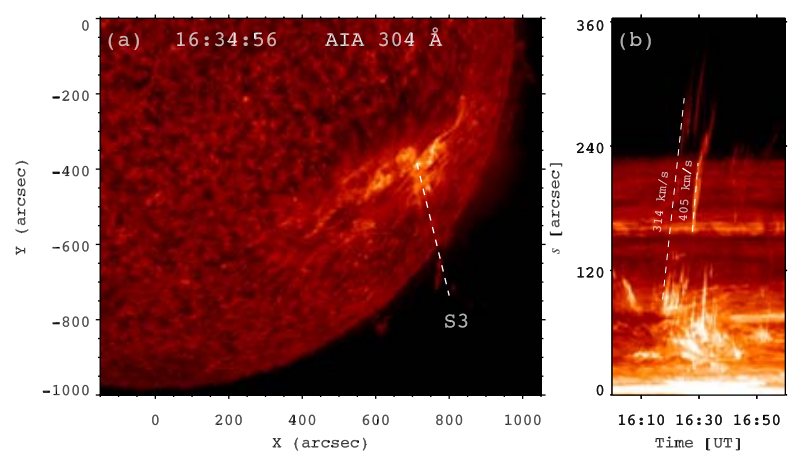

Fig. 6. Panel a: AIA $304 \AA$ image at 16:34:56 UT. Panel $b$ : timedistance diagram of S3 in $304 \AA$. On the $y$-axis, $s=0^{\prime \prime}$ and $s=363^{\prime \prime}$ stand for the northeast and southwest endpoints of S3. The apparent velocities of the outflow are labeled.

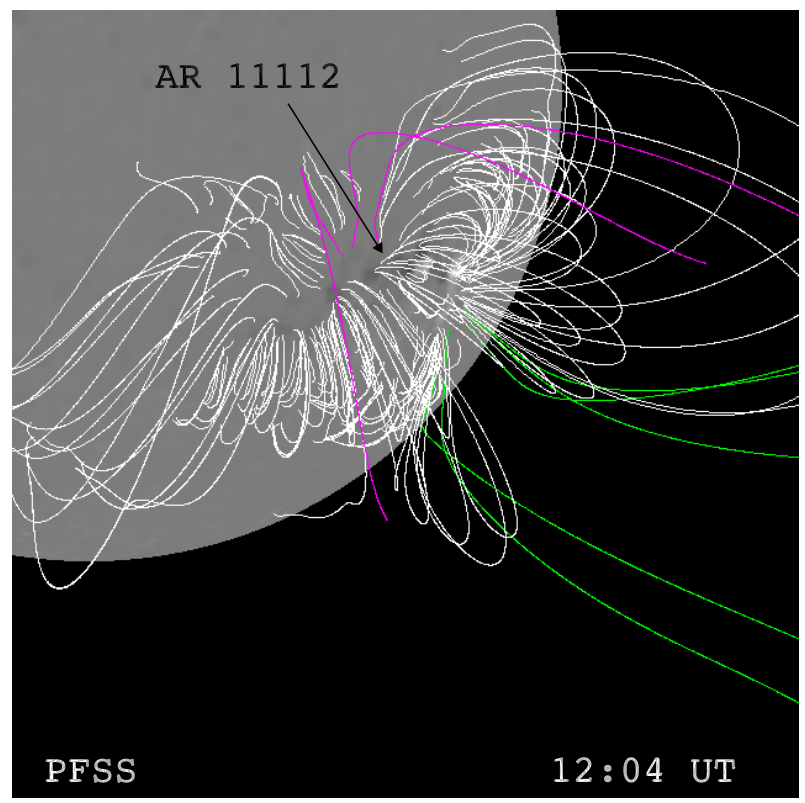

Fig. 7. Three-dimensional magnetic field lines around AR 11112 at 12:04 UT obtained by PFSS modeling. Open and closed field lines are coded with green/magenta and white lines, respectively. The grayscale image denotes the LOS component of the magnetic field at the photosphere.

In Fig. 11 close-ups of the six subregions are shown with a better contrast. To investigate the oscillations we track the oscillating threads and mark their positions manually with white diamonds. The marked positions are independently plotted in Fig. 12. To derive the physical parameters of oscillations, we carry out curve fittings by utilizing a sine function multiplied by an exponential term plus a linear term:

$y=y_{0}+b t+A_{0} \sin \left(\frac{2 \pi}{P} t+\phi_{0}\right) e^{-t / \tau}$

where $y_{0}, A_{0}$, and $\phi_{0}$ represent the initial position, amplitude, and phase, and $b, P$, and $\tau$ stand for the linear velocity, period, and damping time. The parameters are listed in Table 2. According to the pendulum model of longitudinal filament oscillations (Luna \& Karpen 2012; Zhang et al. 2012), the period $(P)$ depends mainly on the curvature radius $(R)$ of a magnetic dip, $P=2 \pi \sqrt{R / g_{\odot}}$, where $g_{\odot}=2.7 \times 10^{2} \mathrm{~m} \mathrm{~s}^{-2}$ is the gravity acceleration of the Sun. In this way, the curvature radius of an oscillating thread can be estimated, $R_{\mathrm{Mm}}=0.025 P_{\mathrm{min}}^{2}$, where the units of $R$ and $P$ are in 


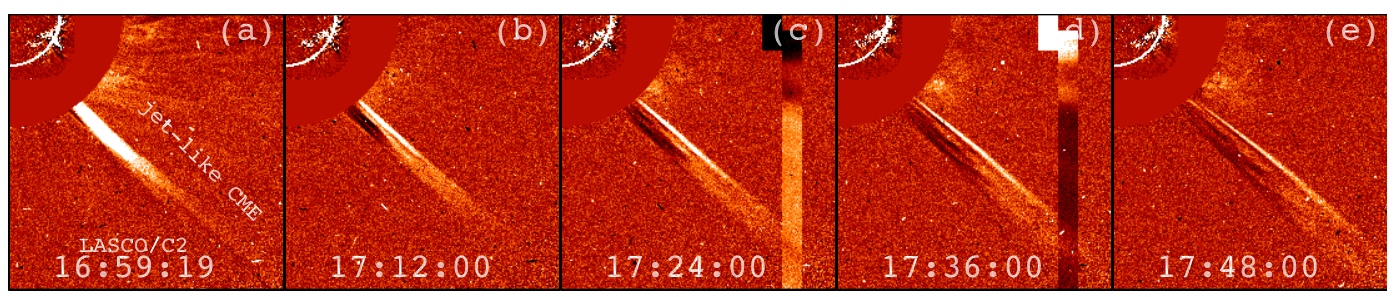

Fig. 8. Running-difference images of the jet-like narrow CME in the field of view of LASCO/C2.

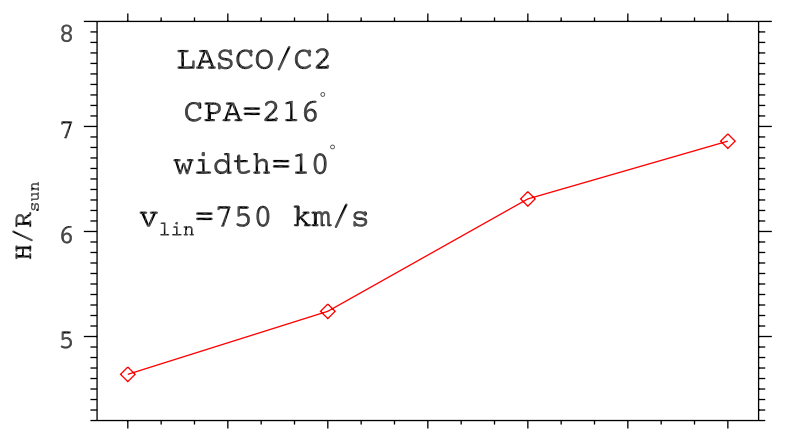

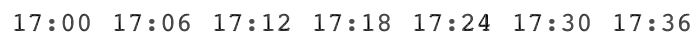

Start Time (18-Oct-10 16:59:06)

Fig. 9. Height-time plot of the jet-like narrow CME. $R_{\text {sun }}$ represents the solar radius. The central position angle (CPA), angular width, and linear velocity of the CME are labeled.

Mm and minute, respectively. The estimated values of $R$ are listed in the last row of Table 2.

From Table 2, it is found that the initial amplitude ranges from 1.6 to $30 \mathrm{Mm}$ with a mean value of $13.8 \mathrm{Mm}$. The amplitudes after flares are considerably larger than those before flares, indicating that the oscillations are greatly enhanced by flares. The initial velocity ranges from $\sim 3$ to $\sim 52 \mathrm{~km} \mathrm{~s}^{-1}$ with a mean value of $\sim 28 \mathrm{~km} \mathrm{~s}^{-1}$. The period ranges from 34 to 73 min with a mean value of $\sim 53 \mathrm{~min}$, which is close to the period of prominence oscillation on 2007 February 8 (Zhang et al. 2012) and periods of filament oscillations on 2010 August 20 (Luna et al. 2014). The curvature radius ranges from $\sim 29$ to $\sim 133 \mathrm{Mm}$ with a mean value of $73.5 \mathrm{Mm}$, implying that the magnetic configurations of oscillating threads differ remarkably (Luna et al. 2014; Zhang et al. 2017b). The oscillations with smaller amplitudes (sub1-sub3) hardly damp with time, while oscillations with larger amplitudes (sub4-sub6) damp quickly. The damping time ranges from $\sim 62$ to $\sim 96 \mathrm{~min}$, with a mean value of $82.4 \mathrm{~min}$. The value of $\tau / P$ has a range of $1.2-1.8$ and a mean value of 1.6, which is comparable to those of longitudinal oscillations on 2015 June 29 (Zhang et al. 2017a), but lower than most of the values (2-4) in the literature (e.g., Jing et al. 2003; Vršnak et al. 2007; Zhang et al. 2012, 2017b; Luna et al. 2017). Observations in this study confirm our previous finding that oscillations with larger initial amplitudes tend to attenuate faster $\left(\tau \sim v_{0}^{-0.3}\right)$ (Zhang et al. 2013).

\section{Numerical simulations}

It is shown in Fig. 10 that solar flares can trigger longitudinal filament oscillations, and can also enhance them. In other words, flares can supply both energy and momentum to the filaments. To reproduce this interesting phenomenon, especially for thd $\mathrm{d}_{a}$, we perform 1D HD numerical simulations following the previous works (Zhang et al. 2012, 2013). The whole evolution of a filament in a flux tube is divided into six steps: formation (or condensation) of cool material after chromospheric evaporation as a result of thermal instability; steady growth due to continuous heating and evaporation at the footpoints; relaxation into a thermal and dynamic equilibrium state after evaporation is halted; oscillation with a smaller amplitude after an unknown small flare; large-amplitude oscillation after flare_1; and oscillation with a larger amplitude after flare_2. First, we briefly introduce the method.

\subsection{Simulation setup}

The 1D HD equations including optically thin radiation are as follows (Xia et al. 2011):

$$
\begin{aligned}
& \frac{\partial \rho}{\partial t}+\frac{\partial}{\partial s}(\rho v)=0, \\
& \frac{\partial}{\partial t}(\rho v)+\frac{\partial}{\partial s}\left(\rho v^{2}+p\right)=\rho g_{\|}(s), \\
& \frac{\partial \varepsilon}{\partial t}+\frac{\partial}{\partial s}(\varepsilon v+p v)=\rho g_{\|} v+H-n_{\mathrm{H}} n_{\mathrm{e}} \Lambda(T)+\frac{\partial}{\partial s}\left(\kappa \frac{\partial T}{\partial s}\right) .
\end{aligned}
$$

Here $\rho, T, p, v, n_{\mathrm{e}}$, and $n_{\mathrm{H}}$ have their normal meanings $(\rho=$ $\left.1.4 m_{\mathrm{p}} n_{\mathrm{H}}, p=2.3 n_{\mathrm{H}} k_{\mathrm{B}} T\right) ; g_{\|}(s)$ is the component of gravity at a distance $(s)$ along the flux tube; $\gamma=5 / 3$ is the ratio of specific heats; $\varepsilon=\rho v^{2} / 2+p /(\gamma-1)$ is the total energy density; $H(s)$ is the volumetric heating rate; $\Lambda(T)$ is the radiative loss function (Rosner et al. 1978; Colgan et al. 2008); and $\kappa=10^{-6} \mathrm{~T}^{5 / 2} \mathrm{ergs} \mathrm{cm}{ }^{-1} \mathrm{~s}^{-1} \mathrm{~K}^{-1}$ is the Spitzer heat conductivity. The above conservative equations are numerically solved using the MPI-AMRVAC ${ }^{2}$ code (Keppens et al. 2012; Porth et al. 2014). The TVDLF scheme is adopted and a five-level AMR is used to produce a highest resolution of $7.7 \mathrm{~km}$.

Figure 13 shows the geometry of symmetric flux tube, consisting of two vertical legs with a length of $h$, two quarter-circular shoulders with a radius of $r$, and a quasi-sinusoidal-shaped dip with a length of $2 w$ and a depth of $D$ (Zhang et al. 2012, 2013). Hence, the total length of the flux tube $L=2 h+\pi r+2 w$. The distribution of $g_{\|}(s)$ along the tube is determined by the geometry. In this study, we take $h=10 \mathrm{Mm}, r=10 \mathrm{Mm}$, and $D=10 \mathrm{Mm}$, so that the magnetic dip has a height of $10 \mathrm{Mm}$ above the photosphere. The total length of dip is $184.68 \mathrm{Mm}$, which corresponds to a curvature radius of $90.1 \mathrm{Mm}$ and an eigen period of $60.03 \mathrm{~min}$. This is equal to the period of thd $\mathrm{d}_{a}$ in sub1.

The first three steps (formation, growth, and relaxation of filament material) are the same as previous works (Xia et al. 2011; Zhang et al. 2013; Zhou et al. 2014). Figure 14 shows the density distribution of the flux tube after reaching an equilibrium state. The filament is located at the bottom of the dip. The initial length, temperature, and density of thread are 12.9 Mm, $1.6 \times 10^{4} \mathrm{~K}$, and $\sim 10^{10} \mathrm{~cm}^{-3}$, respectively. Oscillations

2 http://amrvac.org/index.html 


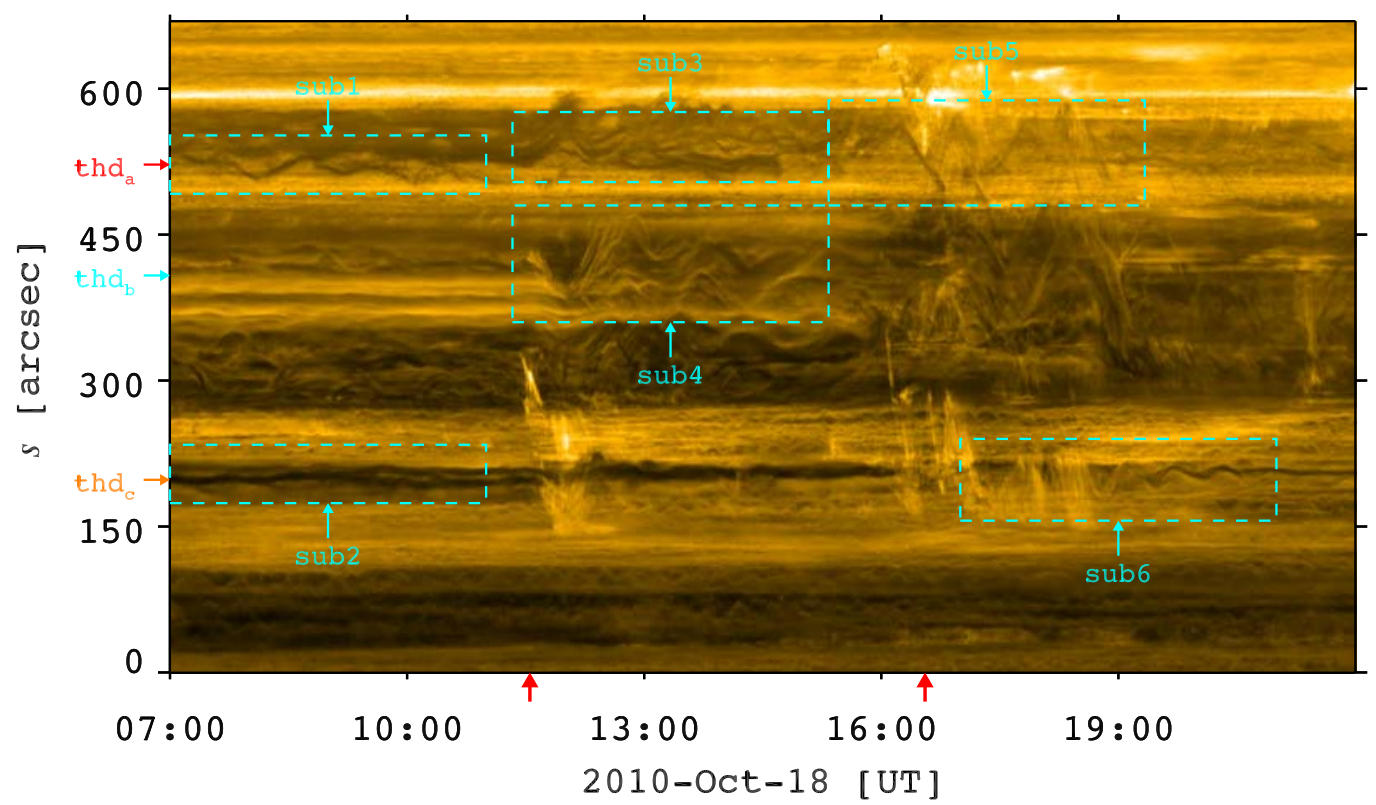

Fig. 10. Time-distance diagram of $\mathrm{S} 1$ in $171 \AA \AA$. On the $y$-axis. $s=0^{\prime \prime}$ and $s=669^{\prime \prime}$ stand for the southeast and northwest endpoints of S1. Horizontal arrows in red, cyan, and orange mark the positions of thd ${ }_{a}$, thd ${ }_{b}$, and thd $\mathrm{d}_{c}$, respectively. Six subregions within the cyan boxes are extracted to explore the longitudinal oscillations of EP. The two vertical arrows under the $x$-axis indicate the times of the flares (11:33 UT and $16: 33$ UT).

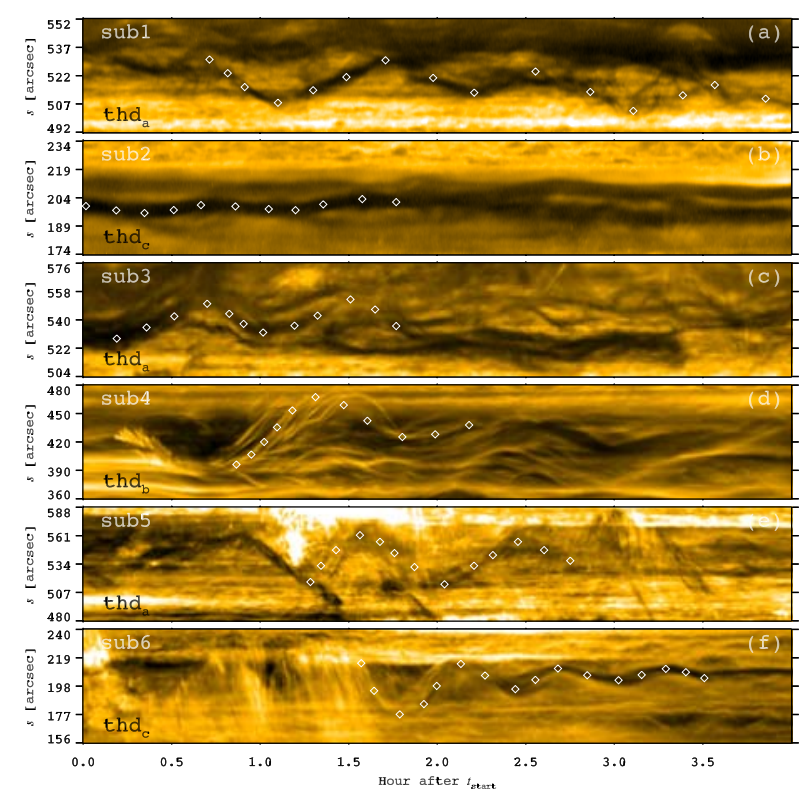

Fig. 11. Close-ups of the six subregions in Fig. 10. White diamonds denote the positions of oscillating threads. The $t_{\text {start }}$ for each subregion is listed in Table 2.

of the thread are divided into three phases according to different impulsive heating rate $H_{\mathrm{f}(\mathrm{s})}$ :

$H_{\mathrm{f}(\mathrm{s})}=E_{2} \exp \left[-\frac{\left(s-s_{\text {peak }}\right)^{2}}{s_{\text {scale }}^{2}}-\frac{\left(t-t_{\text {peak }}\right)^{2}}{t_{\text {scale }}^{2}}\right]$,

with the heating spatial scale $s_{\text {scale }}=2.5 \mathrm{Mm}$, peak location $s_{\text {peak }}=15 \mathrm{Mm}$ (near the left footpoint), and heating timescale $t_{\text {scale }}=5 \mathrm{~min}$. Our simulations are consistent with the observations that the energy deposition (flare ribbons) are near the footpoints of oscillating filament (see Figs. 3 and 4). Impulsive heating, with maximum heating rate $\left(E_{2}\right)$ of $0.015,0.010$, and

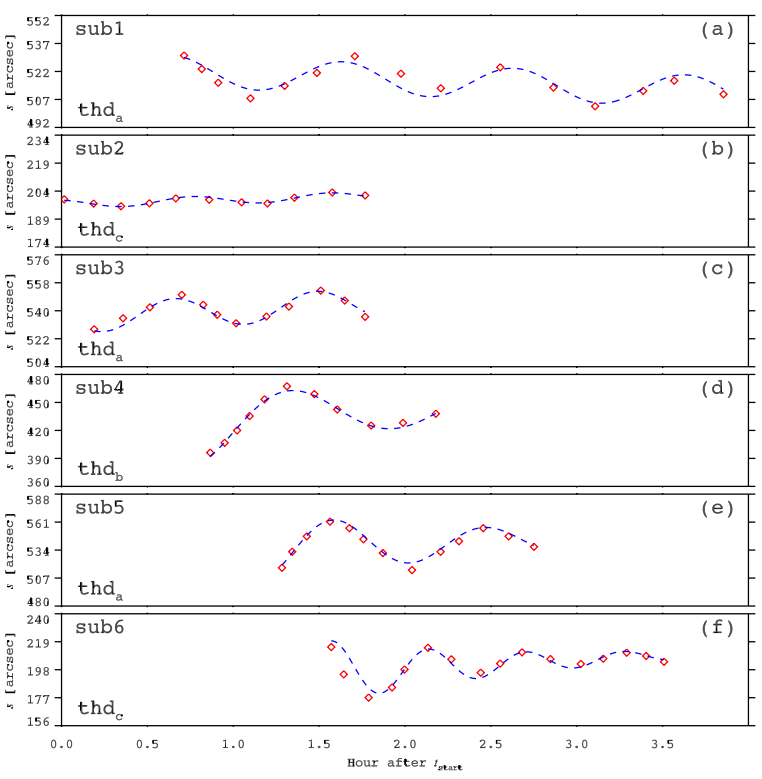

Fig. 12. Marked positions of the oscillating threads (red diamonds) and results of curve fitting (blue lines). The $t_{\text {start }}$ for each subregion is listed in Table 2.

$0.056 \mathrm{erg} \mathrm{cm}^{-3} \mathrm{~s}^{-1}$, is deposited $0.42,3.52$, and $8.52 \mathrm{~h}$ after the equilibrium state $(t=0 \mathrm{~h})$ to mimic a flare (unknown), a C-class flare (flare_1), and another C-class flare (flare_2), respectively.

\subsection{Simulation results}

In Fig. 15, the time evolutions of density and temperature distributions along the flux tube are plotted in the top and bottom panels. In Fig. 16, the time evolution of filament mass center is plotted with black lines and the results of fitting are plotted with colored lines. The evolution of filament oscillation is divided into three phases. In phase I, the filament oscillation is triggered 
Q. M. Zhang et al.: Longitudinal oscillations of an active region filament

Table 2. Parameters of filament oscillations observed by AIA and estimated curvature radii $(R)$ of the threads.

\begin{tabular}{|c|c|c|c|c|c|c|c|}
\hline & Sub1 & Sub2 & Sub3 & Sub4 & Sub5 & Sub6 & Average \\
\hline & $\left(\operatorname{thd}_{a}\right)$ & $\left(\operatorname{thd}_{c}\right)$ & $\left(\operatorname{thd}_{a}\right)$ & $\left(\operatorname{thd}_{b}\right)$ & $\left(\operatorname{thd}_{a}\right)$ & $\left(\operatorname{thd}_{c}\right)$ & \\
\hline$t_{\text {start }}(\mathrm{UT})$ & 07:00 & 07:00 & $11: 20$ & $11: 20$ & $15: 20$ & $19: 20$ & - \\
\hline$t_{\text {end }}(\mathrm{UT})$ & $11: 00$ & $11: 00$ & $15: 20$ & $15: 20$ & $17: 00$ & $21: 00$ & - \\
\hline$A_{0}(\mathrm{Mm})$ & 6.1 & 1.6 & 6.8 & 30.0 & 21.4 & 17.0 & 13.8 \\
\hline$V_{0}\left(\mathrm{~km} \mathrm{~s}^{-1}\right)$ & 10.7 & 3.5 & 14.4 & 43.0 & 42.4 & 52.4 & 27.7 \\
\hline$P(\min )$ & 59.9 & 48.0 & 49.6 & 73.0 & 52.9 & 34.0 & 52.9 \\
\hline$\tau(\min )$ & - & - & - & 88.5 & 96.2 & 62.5 & 82.4 \\
\hline$\tau / P$ & - & - & - & 1.2 & 1.8 & 1.8 & 1.6 \\
\hline$R(\mathrm{Mm})$ & 89.7 & 57.6 & 61.5 & 133.2 & 70.0 & 28.9 & 73.5 \\
\hline
\end{tabular}

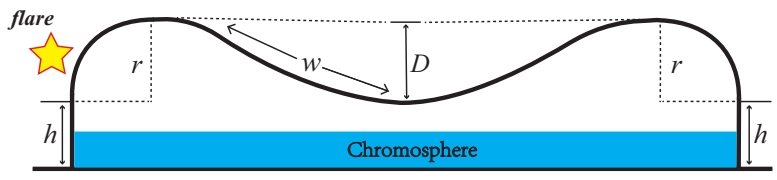

Fig. 13. Geometry of the flux tube used for 1D HD numerical simulations of filament oscillations. See text for details.

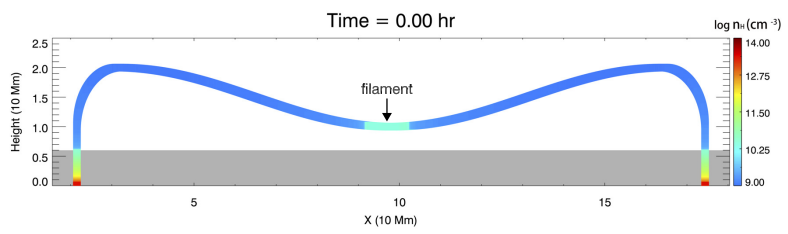

Fig. 14. Density distribution of the flux tube after reaching an equilibrium state. The evolution of flux tube is shown in a movie (anim3.mp4) available online.

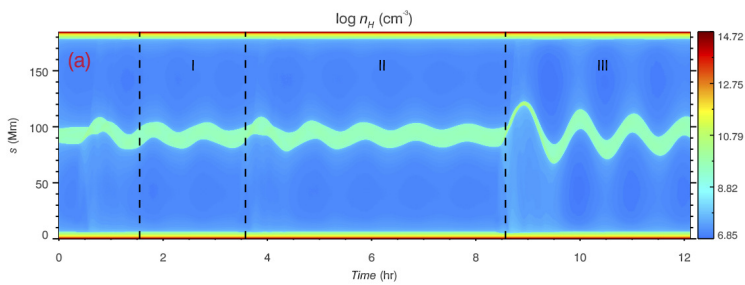

$T$ (MK)

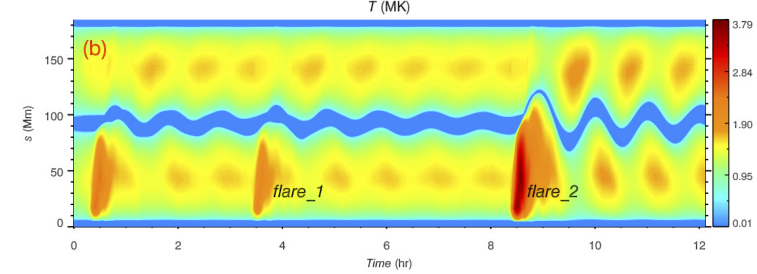

Fig. 15. Time evolutions of density (top panel) and temperature (bottom panel) distributions along the flux tube during filament oscillations. $t=0 \mathrm{hr}$ signifies the start time of the simulation when the flux tube reaches an equilibrium state. The three phases of oscillations are labeled in panel $a$.

by the first impulsive heating resembling an unknown flare and lasts for $\sim 3.1 \mathrm{hrs}$. The initial amplitude, period, and damping time are $5.3 \mathrm{Mm}, 59 \mathrm{~min}$, and $264 \mathrm{~min}$, respectively (see also Table 3 ). The value of $\tau / P$ is close to 4.5 , indicating slow attenuation. The period is in accordance with the period of $\operatorname{thd}_{a}$ in sub1, while the amplitude is slightly lower. In phase II, the oscillation is enhanced by flare_1 and lasts for $\sim 5 \mathrm{hrs}$. The period remains $\sim 60$ min because the geometry of the dip does not change. The

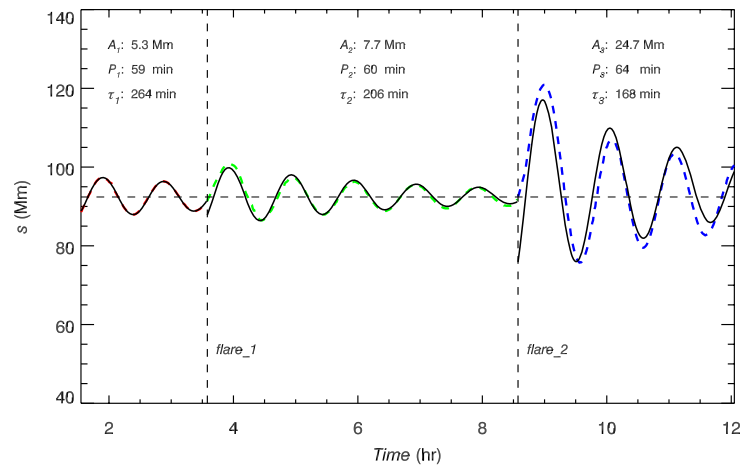

Fig. 16. Time evolution of filament mass center during its oscillations. The black solid line represents the results of simulations. The red, green, and blue lines represent the results of curve fittings. The fitted parameters, including initial amplitudes, periods, and damping times, are labeled.

Table 3. Parameters of simulated filament oscillations during the three phases.

\begin{tabular}{lccc}
\hline \hline Phase & I & II & III \\
\hline$\Delta t(\mathrm{hr})$ & 3.1 & 5.0 & 3.6 \\
$A_{0}(\mathrm{Mm})$ & 5.3 & 7.7 & 24.7 \\
$P(\min )$ & 59 & 60 & 64 \\
$\tau(\min )$ & 264 & 206 & 168 \\
$\tau / P$ & 4.5 & 3.4 & 2.6 \\
\hline
\end{tabular}

initial amplitude increases to $7.7 \mathrm{Mm}$, which is larger than that of thd ${ }_{a}$ after the C1.3 flare in sub3. The damping time decreases, indicating faster attenuation $(\tau / P \approx 3.4)$. In phase III, the oscillation is enhanced by flare_2 and lasts for $3.6 \mathrm{hrs}$. The amplitude increases significantly to $24.7 \mathrm{Mm}$, which is comparable to that of thd $\mathrm{d}_{a}$ after the C2.6 flare in sub5. The period increases slightly to $64 \mathrm{~min}$, which is predicted by the previous simulations that the period increases marginally with the initial amplitude $\left(P \sim v_{0}^{0.05}\right)$ when the geometry is fixed (Zhang et al. 2013). The damping time decreases to $168 \mathrm{~min}$ and $\tau / P$ decreases to 2.6. It is obvious that our simulations can satisfactorily reproduce the longitudinal oscillations triggered and enhanced by solar flares. Both the amplitudes and periods are roughly in agreement with the results of thd $\mathrm{d}_{a}$. It should be noted that the slow attenuation in phases I and II agrees with the situation of thd $\mathrm{d}_{a}$ before the C2.6 flare, while the damping time in phase III is still insufficient to explain the quick damping of thd ${ }_{a}$ after the C2.6 flare. Since the dominant damping mechanism of longitudinal filament oscillations in 
our model is radiative loss (Zhang et al. 2013), a combination of radiative loss and wave leakage may be helpful in interpreting the observed quick attenuation (Zhang et al. 2019).

\section{Discussion}

\subsection{Relationship between flares and filament oscillations}

Since the first discovery of longitudinal filament oscillations (Jing et al. 2003), the triggering mechanisms have been extensively investigated, such as microflares (Jing et al. 2006; Vršnak et al. 2007; Zhang et al. 2012, 2017b), coronal jets (Luna et al. 2014; Zhang et al. 2017a), shock waves (Shen et al. 2014a; Pant et al. 2016), merging of two solar filaments (Luna et al. 2017), and failed filament eruption (Mazumder et al. 2020). Longitudinal filament oscillations triggered by flares have been well established (Luna \& Karpen 2012; Zhang et al. 2013; Zhou et al. 2017). However, filament oscillations enhanced by flares have never been reported. In this paper, for the first time, we report longitudinal filament oscillations enhanced by two C-class flares in AR 11112. The amplitudes increased from 6.1 Mm to $6.8 \mathrm{Mm}$ after the $\mathrm{C} 1.3$ flare and further to $21.4 \mathrm{Mm}$ after the $\mathrm{C} 2.6$ flare, with the period variation being $\leq 20 \%$. The roles of flares are additionally confirmed by 1D HD numerical simulations based on the previous works. The simulated amplitudes and periods are close to the observed values, while the damping time in the last phase is longer than the observed value, implying additional mechanisms may play a role. Our findings can be interpreted as the daily experience of playing on a swing. Before the swing comes to a halt, the amplitude is amplified when new momentum is deposited, so that the oscillation is extended dramatically.

\subsection{Relationship between filament oscillations and solar eruptions}

The relationship between large-amplitude filament oscillations and solar eruptions is still unclear. On one hand, coronal EUV waves associated with CMEs and Moreton waves associated with flares can trigger filament oscillations (e.g., Eto et al. 2002; Dai et al. 2012; Liu et al. 2013). On the other hand, after studying the transverse oscillation of a prominence using the spectroscopic observation from SOHO/SUMER, Chen et al. (2008) proposed that transverse filament oscillation can serve as another precursor of CMEs, which is supported by state-of-the-art observations from IRIS (Zhou et al. 2016). After studying the longitudinal oscillation of a prominence on 2007 February 8, Zhang et al. (2012) proposed that longitudinal filament oscillation can serve as a new precursor of flares and CMEs, which is supported by the imaging observations from SDO/AIA (Bi et al. 2014).

In this study the filament was divided into two parts, the EP and WP. Only the EP oscillated, lasting for about $14 \mathrm{hrs}$. Both parts survived the $\mathrm{C} 1.3$ flare, while the WP erupted and produced a C2.6 flare and a jet-like CME. Since the two parts are close to each other, it is likely that they have interplay during the evolution. Longitudinal oscillations of the EP may stimulate the destabilization and final eruption of the WP. Compression from the overlying magnetic field above the EP was strong enough to prevent it from eruption after the C2.6 flare. It should be noted that we did not perform a nonlinear force free field extrapolation, since the AR was very close to the limb.

\section{Summary}

In this paper we investigate large-amplitude, longitudinal oscillations of the EP of a very long filament in AR 11112 observed by SDO/AIA and GONG on 2010 October 18. HD numerical simulations using the MPI-AMRVAC code are conducted to reproduce part of the observations. The main results are summarized as follows:

1. During the evolution of filament, two homologous flares occurred in the same AR. The C1.3 flare was confined without a CME. Both EP and WP of the filament were slightly disturbed and survived the flare. After $5 \mathrm{hrs}$, eruption of the WP generated a C2.6 flare and a narrow, jet-like CME observed by LASCO/C2.

2. Three oscillating threads $\left(\operatorname{thd}_{a}, \operatorname{thd}_{b}\right.$, and thd $\mathrm{d}_{c}$ ) are clearly identified in the EP and their oscillations are naturally divided into three phases by the two flares. The initial amplitude ranges from 1.6 to $30 \mathrm{Mm}$ with a mean value of $\sim 14 \mathrm{Mm}$. The period ranges from 34 to 73 min with a mean value of $\sim 53 \mathrm{~min}$. The curvature radii of the magnetic dips are estimated to be 29 to $133 \mathrm{Mm}$ with a mean value of $\sim 74 \mathrm{Mm}$. The damping time ranges from $\sim 62$ to $\sim 96 \mathrm{~min}$ with a mean value of $\sim 82 \mathrm{~min} . \tau / P$ is between 1.2 and 1.8 .

3. For thd $\mathrm{d}_{a}$ in the EP, the amplitudes were enhanced by the two flares from $6.1 \mathrm{Mm}$ to $6.8 \mathrm{Mm}$ after the $\mathrm{C} 1.3$ flare and further to $21.4 \mathrm{Mm}$ after the $\mathrm{C} 2.6$ flare. The period variation as a result of perturbation from the flares was $\leq 20 \%$. The attenuation became faster after the $\mathrm{C} 2.6$ flare. To the best of our knowledge, this is the first report of large-amplitude, longitudinal filament oscillations enhanced by flares.

4. Numerical simulations reproduce the oscillations of $\operatorname{thd}_{a}$ very well. The simulated amplitudes and periods are close to the observed values, while the damping time in the last phase is longer, implying additional mechanisms should be taken into account apart from radiative loss.

Acknowledgements. The authors thank the referee for valuable suggestions and comments to improve the quality of this article. SDO is a mission of NASA's Living With a Star Program. AIA and HMI data are courtesy of the NASA/SDO science teams. This work utilizes GONG data from NSO, which is operated by AURA under a cooperative agreement with NSF and with additional financial support from NOAA, NASA, and USAF. This work is funded by NSFC grants (No. 11773079, 11790302), the Science and Technology Development Fund of Macau (275/2017/A), the International Cooperation and Interchange Program (11961131002), the Youth Innovation Promotion Association CAS, and the project supported by the Specialized Research Fund for State Key Laboratories. J.H.G is also supported by the Chinese NSFC grant 11533005 .

\section{References}

Arregui, I., \& Ballester, J. L. 2011, Space Sci. Rev., 158, 169

Arregui, I., Oliver, R., \& Ballester, J. L. 2012, Liv. Rev. Solar Phys., 9, 2 Awasthi, A. K., Liu, R., \& Wang, Y. 2019, ApJ, 872, 109

Berger, T. E., Slater, G., Hurlburt, N., et al. 2010, ApJ, 716, 1288

Bi, Y., Jiang, Y., Yang, J., et al. 2014, ApJ, 790, 100

Brueckner, G. E., Howard, R. A., Koomen, M. J., et al. 1995, Sol. Phys., 162, 357

Chen, P. F. 2011, Liv. Rev. Solar Phys., 8, 1

Chen, P. F., Innes, D. E., \& Solanki, S. K. 2008, A\&A, 484, 487

Chen, P. F., Harra, L. K., \& Fang, C. 2014, ApJ, 784, 50

Colgan, J., Abdallah, Jr., J., Sherrill, M. E., et al. 2008, ApJ, 689, 585

Dai, Y., Ding, M. D., Chen, P. F., \& Zhang, J. 2012, ApJ, 759, 55

DeVore, C. R., \& Antiochos, S. K. 2000, ApJ, 539, 954

Engvold, O. 1976, Sol. Phys., 49, 283

Eto, S., Isobe, H., Narukage, N., et al. 2002, PASJ, 54, 481

Fletcher, L., Dennis, B. R., Hudson, H. S., et al. 2011, Space Sci. Rev., 159, 19

Gibson, S. E. 2018, Liv. Rev. Solar Phys., 15, 7

Gunár, S., \& Mackay, D. H. 2015, ApJ, 803, 64

Hyder, C. L. 1966, Z. Astrophys., 63, 78

Ji, H., Wang, H., Schmahl, E. J., et al. 2003, ApJ, 595, L135

Jing, J., Lee, J., Spirock, T. J., et al. 2003, ApJ, 584, L103

Jing, J., Lee, J., Spirock, T. J., \& Wang, H. 2006, Sol. Phys., 236, 97

Keppens, R., \& Xia, C. 2014, ApJ, 789, 22 
Q. M. Zhang et al.: Longitudinal oscillations of an active region filament

Keppens, R., Meliani, Z., van Marle, A. J., et al. 2012, J. Comput. Phys., 231, 718

Kleczek, J., \& Kuperus, M. 1969, Sol. Phys., 6, 72

Labrosse, N., Heinzel, P., Vial, J.-C., et al. 2010, Space Sci. Rev., 151, 243

Lemen, J. R., Title, A. M., Akin, D. J., et al. 2012, Sol. Phys., 275, 17

Li, T., \& Zhang, J. 2012, ApJ, 760, L10

Li, T., \& Zhang, J. 2013, ApJ, 770, L25

Li, L., Zhang, J., Peter, H., et al. 2018a, ApJ, 864, L4

Li, D., Shen, Y., Ning, Z., et al. 2018b, ApJ, 863, 192

Lin, Y., Engvold, O., Rouppe van der Voort, L. H. M., \& van Noort, M. 2007, Sol. Phys., 246, 65

Lites, B. W. 2005, ApJ, 622, 1275

Liu, R., Kliem, B., Török, T., et al. 2012, ApJ, 756, 59

Liu, R., Liu, C., Xu, Y., et al. 2013, ApJ, 773, 166

Luna, M., \& Karpen, J. 2012, ApJ, 750, L1

Luna, M., Díaz, A. J., \& Karpen, J. 2012, ApJ, 757, 98

Luna, M., Knizhnik, K., Muglach, K., et al. 2014, ApJ, 785, 79

Luna, M., Terradas, J., Khomenko, E., Collados, M., \& de Vicente, A. 2016, ApJ, 817,157

Luna, M., Su, Y., Schmieder, B., et al. 2017, ApJ, 850, 143

Luna, M., Karpen, J., Ballester, J. L., et al. 2018, ApJS, 236, 35

Mackay, D. H., Karpen, J. T., Ballester, J. L., Schmieder, B., \& Aulanier, G. 2010, Space Sci. Rev., 151, 333

Martens, P. C., \& Zwaan, C. 2001, ApJ, 558, 872

Martin, S. F. 1998, Sol. Phys., 182, 107

Mazumder, R., Pant, V., Luna, M., et al. 2020, A\&A, 633, A12

Ning, Z., Cao, W., Okamoto, T. J., Ichimoto, K., \& Qu, Z. Q. 2009, A\&A, 499, 595

Oliver, R., \& Ballester, J. L. 2002, Sol. Phys., 206, 45

Pant, V., Mazumder, R., Yuan, D., et al. 2016, Sol. Phys., 291, 3303

Parenti, S. 2014, Liv. Rev. Solar Phys., 11, 1

Porth, O., Xia, C., Hendrix, T., et al. 2014, ApJS, 214, 4

Ramsey, H. E., \& Smith, S. F. 1966, AJ, 71, 197

Rosner, R., Tucker, W. H., \& Vaiana, G. S. 1978, ApJ, 220, 643

Ruderman, M. S., \& Luna, M. 2016, A\&A, 591, A131
Scherrer, P. H., Schou, J., Bush, R. I., et al. 2012, Sol. Phys., 275, 207 Schmieder, B., Chandra, R., Berlicki, A., et al. 2010, A\&A, 514, A68 Schmieder, B., Tian, H., Kucera, T., et al. 2014, A\&A, 569, A85 Schrijver, C. J., \& De Rosa, M. L. 2003, Sol. Phys., 212, 165

Shen, Y., Liu, Y. D., Chen, P. F., \& Ichimoto, K. 2014a, ApJ, 795, 130

Shen, Y., Ichimoto, K., Ishii, T. T., et al. 2014b, ApJ, 786, 151

Shen, Y., Liu, Y., Liu, Y. D., et al. 2015, ApJ, 814, L17

Shibata, K., Masuda, S., Shimojo, M., et al. 1995, ApJ, 451, L83

Su, Y., \& van Ballegooijen, A. 2012, ApJ, 757, 168

van Ballegooijen, A. A. 2004, ApJ, 612, 519

Vial, J.-C., \& Engvold, O. 2015, Solar Prominences (Springer International Publishing)

Vršnak, B., Veronig, A. M., Thalmann, J. K., \& Žic, T. 2007, A\&A, 471, 295

Woods, T. N., Eparvier, F. G., Hock, R., et al. 2012, Sol. Phys., 275, 115

Xia, C., Chen, P. F., Keppens, R., et al. 2011, ApJ, 737, 27

Xia, C., Chen, P. F., \& Keppens, R. 2012, ApJ, 748, L26

Yan, X. L., Xue, Z. K., Pan, G. M., et al. 2015, ApJS, 219, 17

Yang, L., Yan, X., Li, T., et al. 2017, ApJ, 838, 131

Zapiór, M., Schmieder, B., Mein, P., et al. 2019, A\&A, 623, A144

Zhang, Q. M., \& Ji, H. S. 2014, A\&A, 567, A11

Zhang, Q. M., \& Ji, H. S. 2018, ApJ, 860, 113

Zhang, Q. M., Chen, P. F., Xia, C., \& Keppens, R. 2012, A\&A, 542, A52

Zhang, Q. M., Chen, P. F., Xia, C., Keppens, R., \& Ji, H. S. 2013, A\&A, 554, A124

Zhang, Q. M., Ning, Z. J., Guo, Y., et al. 2015, ApJ, 805, 4

Zhang, Q. M., Li, D., \& Ning, Z. J. 2017a, ApJ, 851, 47

Zhang, Q. M., Li, T., Zheng, R. S., Su, Y. N., \& Ji, H. S. 2017b, ApJ, 842, 27

Zhang, L. Y., Fang, C., \& Chen, P. F. 2019, ApJ, 884, 74

Zhou, Y.-H., Chen, P.-F., Zhang, Q.-M., et al. 2014, Res. Astron. Astrophys., 14, 581

Zhou, G. P., Zhang, J., \& Wang, J. X. 2016, ApJ, 823, L19

Zhou, Y.-H., Zhang, L.-Y., Ouyang, Y., Chen, P. F., \& Fang, C. 2017, ApJ, 839, 9

Zhou, Y.-H., Xia, C., Keppens, R., et al. 2018, ApJ, 856, 179 